\title{
Cuantificación del ahorro de energía eléctrica en clientes residenciales mediante acciones de gestión de demanda
}

\section{Quantification of electrical energy savings in residential customers through demand management strategies}

\author{
Orlando González ${ }^{1}$, Andrés Pavas², Samir Sánchez ${ }^{3}$ \\ ${ }^{1}$ Programa de investigacion sobre adquisicion y analisis de señales PAAS-UN, Departamento de Ingeniería Eléctrica y \\ Electrónica, Universidad Nacional, Colombia. Email: oagonzalezv@unal.edu.co \\ ${ }^{2}$ Programa de investigacion sobre adquisicion y analisis de señales PAAS-UN, Departamento de Ingeniería Eléctrica y \\ Electrónica, Universidad Nacional, Colombia. Email: fapavasm@unal.edu.co \\ ${ }^{3}$ Programa de investigacion sobre adquisicion y analisis de señales PAAS-UN, Departamento de Ingeniería Eléctrica y \\ Electrónica, Universidad Nacional, Colombia. Email: yssanchezr@ unal.edu.co
}

RECIBIDO: Marzo 20, 2016. ACEPTADO: Febrero 1, 2017. VerSIÓn FInAL: Mayo 15, 2017.

\begin{abstract}
RESUMEN
Colombia enfrentó entre 2015 y 2016 uno de los fenómenos de El Niño más severos de su historia quedando ad-portas de un racionamiento de energía. La gestión de demanda puede ayudar, entre otros aspectos, a reducir el consumo de energía en cualquier escenario de disponibilidad de recursos energéticos, no sólo en emergencia. El artículo presenta la cuantificación de los posibles ahorros en clientes residenciales en Bogotá D.C., determinados a partir del modelado de diferentes estrategias de gestión de demanda. Los resultados muestran que pueden lograrse reducciones en el consumo entre 6 y $26 \%$ mediante sustitución de dispositivos, 7 y $19 \%$ mediante cambio de hábitos de consumo, y entre $17 \%$ y $45 \%$ como ahorro total posible. Los resultados también revelan que los clientes con menores consumos de energía pueden alcanzar ahorros de hasta el $45 \%$, mientras que los clientes con consumos mayores de $150 \mathrm{kWh} / \mathrm{mes}$ no logran ahorros superiores al $20 \%$.
\end{abstract}

PALABRAS ClAVE: Ahorro de energía, Gestión de demanda, Estrategias de gestión de demanda, Modelado de perfiles de carga.

\begin{abstract}
Colombia faced from 2015 to 2016 one of most severe El Niño whether phenomenal in its history, being close to energy rationing. Demand management can help to reduce energy consumption under any scenario of energy availability, not only under emergencies. This article presents the quantification of potential savings in residential customers in Bogotá D.C., determined from the modeling of different demand management strategies. The results show energy reduction between 6 and 26\% resorting to device substitution, 7 and 19\% through energy consumption patterns adjustments, and between $17 \%$ and $45 \%$ as possible total energy savings. The results reveal as well that customers with low energy consumption can reach upto $45 \%$ savings, in contrast to customers with consumption energy greater than $150 \mathrm{kWh} /$ month cannot reach energy saving above $20 \%$.
\end{abstract}

KEYWORDS: Demand management, Demand management strategies, Energy savings, Load profile modeling.

Este artículo puede compartirse bajo la licencia CC BY-ND 4.0 y se referencia usando el siguiente formato: O. González, A. Pavas, S. Sánchez, "Cuantificación del ahorro de energía eléctrica en clientes residenciales mediante acciones de gestión de demanda", UIS Ingenierías, vol. 16, no. 2, pp. 217 - 226, Julio - Diciembre 2017. Doi: https://doi.org/10.18273/revuin.v16n2-2017020 


\section{INTRODUCCIÓN}

Los sistemas eléctricos de potencia alrededor del mundo están experimentando un periodo de rápida evolución. La adopción de la generación distribuida, la incorporación de fuentes renovables de energía, los esquemas de mercados eléctricos competitivos, el almacenamiento de energía, y la respuesta a la demanda estarán presentes en los sistemas eléctricos del futuro [1]. Estos cambios en los sistemas eléctricos de potencia representan no solo retos de ingeniería sino que también cambios para el usuario final [2].

El cambio intencional del perfil de consumo de potencia eléctrica por parte del usuario final en respuesta a un estímulo externo, generalmente de tipo económico, se conoce como respuesta a la demanda y ha sido usado de distas formas en el suministro de energía desde hace ya varias décadas.

La implementación de la respuesta a la demanda plantea principalmente dos desafíos. El primero de ellos de tipo técnico - económico, pues se requieren grandes inversiones en infraestructura que permita la comunicación, control y monitorización del sistema. El segundo está relacionado con el diseño del mercado, además, la respuesta a la demanda requiere coordinación entre un gran número de consumidores, generadores, operadores del sistema de transmisión, distribución, y demás agentes distribuidos a lo largo del sistema.

Una descripción general del desarrollo en respuesta a la demanda se presenta en [3] y en [4]. Un análisis costobeneficio al implementar respuesta a la demanda se encuentra en [5] y en [3], donde se hace una análisis de costos asociados al consumidor y costos debidos la implementación de una infraestructura de comunicación.

La experiencia en respuesta a la demanda en la Unión Europea se discute en [4], donde se evidencia la carencia de políticas que la promuevan. Dentro de los mayores obstáculos para el establecimiento de políticas que permitan apoyar el desarrollo de la respuesta a la demanda se consideran: la incertidumbre que rodea a los verdaderos beneficios y costos de la respuesta a la demanda, la forma de integración dentro de un mercado eléctrico competitivo, y la interacción con otros métodos de gestión en eficiencia energética entre otras.

Particularmente, se ha demostrado que las cargas residenciales juegan un papel importante en el corrimiento y reducción del pico de demanda [6]. Los hábitos de consumo de energía por parte de los usuarios es un factor adicional a tener en cuenta y que introduce un alto grado de dificultad a la hora de diseño de un mercado. Mientras que los grandes generadores suelen exhibir un comportamiento racional, los clientes más pequeños no muestran la misma racionalidad en sus decisiones de consumo, los usuarios finales, especialmente aquellos que pertenecen al sector residencial, tienen diferentes prioridades, dentro de las cuales no se encuentra reducir al mínimo su factura de consumo de energía eléctrica.

La curva de demanda de un usuario residencial es difícil de extraer, pues depende de distintos factores variables en el tiempo, que van desde el clima, hasta el tipo de electrodoméstico usado para realizar la cocción de los alimentos, por ejemplo: horno eléctrico o estufa a gas. En [7] se presenta un estudio sobre los patrones de consumo de dos grupos residenciales, para los cuales se aplican distintos precios de acuerdo al consumo de energía eléctrica, se determinó que el cambio en ese consumo no está linealmente relacionado con el cambio de precios. Los consumidores mostraron una escasa reducción de la demanda durante los periodos de precios pico, pero un aumento significativo en consumo durante los periodos de menor precio.

Un estudio de características similares se presenta en [8] para clientes residenciales en Chicago. Se observó una reducción substancial del consumo durante periodos de precios pico, pero no un incremento durante el periodo de bajo precio. La primera aproximación de gestión de demanda sobre tres electrodomésticos se presenta en [9], el documento usa la estrategia de cambio de hábitos de consumo con el fin de hallar los valores máximos de ahorro de electricidad con una mínima inversión sobre los dispositivos.

En [10], [11] y [12] el actor principal en respuesta a la demanda es el usuario final. En [11] se introdujo una función de demanda que permite representar la demanda de diferentes tipos de cliente, además, de un modelo integral de respuesta de los consumidores ante variaciones de precios. En [10] se propone un modelo óptimo de respuesta a la demanda, que maximiza el beneficio para el proveedor, al tiempo que minimiza el consumo diario de energía eléctrica por parte del usuario final como respuesta a la tarifa diferenciada.

De la anterior revisión es evidente que el comportamiento de los consumidores no se puede abordar a través de modelos económicos convencionales. Además, la gran mayoría de los clientes residenciales tienen poco o ningún conocimiento de los mercados eléctricos, e inclusive de su propio consumo [13]. La interacción del consumidor podría ser realizable fijando hábitos de uso de los electrodomésticos, por ejemplo, fijando la temperatura de la nevera, esto permite entre otras cosas, determinar un perfil óptimo de consumo en respuesta a las señales de precios [2]. 
La obsolescencia tecnológica y el uso de dispositivos eléctricos y electrónicos no eficientes en iluminación, entretenimiento y climatización, posibilitan hacer gestión de demanda a través de la sustitución de estos hacia tecnologías más modernas y eficientes, de acuerdo con [14], la iluminación constituye un componente importante del consumo total de energía. El presente estudio se realizó para la población de estrato IV en la ciudad de Bogotá D.C., para la cual se considera gestión de la demanda con mínima intervención técnica a través de cambios en los hábitos de consumo o gestión de la demanda por medio de sustitución tecnológica.

En la Tabla 1 se presenta un resumen de las cantidades propuestas en la composición de carga residencial. Los resultados de la simulación evidencian un ahorro significativo en el consumo de energía $(\mathrm{kWh} / \mathrm{mes})$ a la hora de realizar sustitución tecnológica en iluminación, especialmente cuando se pasa de una tecnología Incandescente a una de tipo LED.

El artículo está organizado en tres secciones principales. En la Sección 2 se presenta el modelado de los clientes de la electricidad y las estrategias de gestión de demanda propuestas. La Sección 3 se presenta los resultados obtenidos y su análisis. Finalmente, en la Sección 4 se presentan las conclusiones.

\section{METODOLOGÍA}

El procedimiento adoptado para el desarrollo de la hipótesis planteada se presenta a través de tres pasos progresivos, luego de definir las condiciones iniciales del modelo.

\subsection{Condiciones iniciales del modelo}

Población: Usuarios residenciales estrato IV ciudad de Bogotá D.C. La función de distribución para su población dividido en deciles, contiene el valor promedio de la energía consumida mensual para cada grupo de usuarios y se describe en la Fig. 1.

Tipos de carga: Se seleccionaron tres cargas de acuerdo con los requerimientos y necesidades, estas conforman el perfil de demanda:
- Iluminación
- Refrigeración
- Tv junto con Decodificador
- Carga Residual

La cantidad de elementos para conformar la carga se encuentra en la Tabla 1, donde se han determinado los siguientes criterios:
Iluminación es inherente al consumo de electricidad, por tanto, se induce un mínimo de 3 luminarias (baño, habitación y sala) y un máximo de 12 (3 habitaciones, 2 baños, 1 cocina, 1 comedor, 1 sala, 1 patio, 1 cobertizo, 1 corredor y 1 escalera).

El uso de televisión es dispensable para ciertos usuarios por tanto se consideró desde la no existencia, mínimo 0 , hasta un máximo 3 televisores por usuario. Por otra parte aunque se tiene en cuenta la gran penetración de las tecnologías LCD y LED - OLED, aún los CRT se usan en una baja proporción.

En cuanto a decodificadores la mayoría de planes incluyen dos decodificadores sin recargo económico, así se tuvo en cuenta la existencia desde 0 hasta 2 decodificadores por usuario.

La nevera a pesar de ser el elemento de mayor consumo energético, no está presente en todos los hogares de los usuarios, se tuvo en cuenta un rango de 0 a 2 neveras por usuario.

La carga residual se asume como un agregado de los demás elementos o electrodomésticos que no se tienen en cuenta anteriormente.

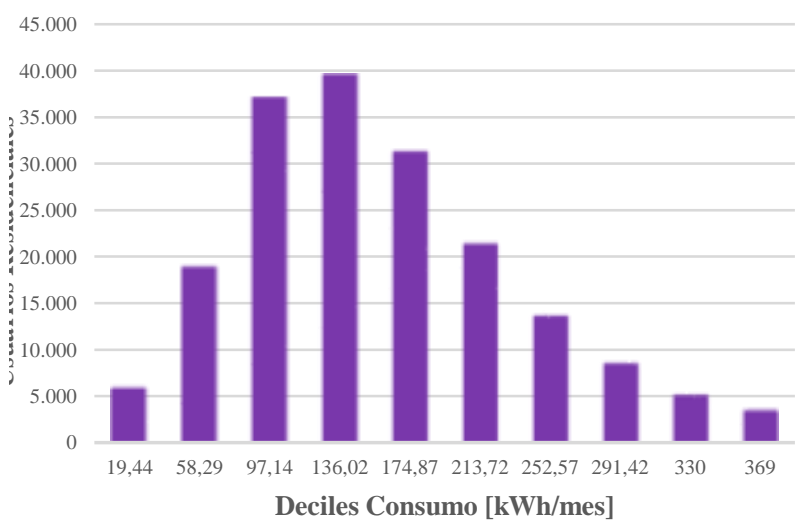

Figura 1. Distribucion de consumo promedio $\mathrm{kWh} / \mathrm{mes}$ para estrato IV en Bogotá D.C. Fuente: [9].

Tabla 1. Conformación de la carga para estrato IV.

\begin{tabular}{|c|c|c|}
\hline \multirow{2}{*}{ Electrodoméstico } & \multicolumn{2}{|c|}{ Cantidad } \\
\cline { 2 - 3 } & Mínima & Máxima \\
\hline Iluminación & 3 & 12 \\
\hline Televisores & 0 & 3 \\
\hline Decodificador & 0 & 2 \\
\hline Neveras & 0 & 2 \\
\hline
\end{tabular}

Fuente. Elaboración propia. 
A cada elemento y tipo de tecnologia se le ha asociado una potencia respectiva, valores tomados de [14] y [15], el tipo de electrodomestico, tecnologia y potencia promedio asociada se presentan en la Tabla 2.

Tabla 2. Clasificación de los electrodomésticos por tecnología.

\begin{tabular}{|c|c|c|}
\hline \multirow{2}{*}{ Elemento } & Tecnología & Potencia [kW] \\
\hline \multirow{2}{*}{ Iluminación } & $\begin{array}{c}\text { Incan- Halógena } \\
\text { CFL } \\
\text { LED }\end{array}$ & 0,60 \\
\cline { 2 - 3 } & CRT & 0,20 \\
\hline \multirow{2}{*}{ Televisores } & LCD & 0,10 \\
\cline { 2 - 3 } & Activo & 0,07 \\
\hline \multirow{2}{*}{ Decodificador } & Stand-by & 0,02 \\
\cline { 2 - 3 } & &
\end{tabular}

Fuente: [14]-[16].

Para el caso de la nevera, se consideró tanto la eficiencia como la temperatura del termostato, los valores se resumen en la Tabla 3. En el tema de eficiencia se toma en cuenta la norma NTC 5020, para un refrigerador sin escarcha y congelador superior de 250 lts (tamaño promedio).

Tabla 3. Clasificación nevera: Temperatura y eficiencia.

\begin{tabular}{|l|c|c|}
\hline Elemento & $\begin{array}{c}\text { Temperatura } \\
{\left[{ }^{\circ} \mathbf{C}\right]}\end{array}$ & $\begin{array}{c}\text { Eficiencia } \\
{[\mathbf{k W h} / \mathbf{m e s}]} \\
\text { Volumen 250 lts }\end{array}$ \\
\hline & 2 & $\mathrm{~A}=25.8$ \\
Nevera & 4 & $\mathrm{D}=36.6$ \\
& 6 & $\mathrm{G}=48.4$ \\
\hline
\end{tabular}

Fuente: [17]y [18].

\subsection{Estrategias de Gestión}

A continuación se enuncian las herramientas de gestión de demanda que se usaron para el desarrollo de este artículo.

\subsubsection{Sustitución de Tecnología.}

Se entiende por sustitucion tecnologica el paso de una tenologia de menor eficiencia energetica a otra de mayor eficiencia, mejorando o manteniendo las caracteristicas del servicio.

Dentro de la estrategia de sustitución se adoptaron las siguientes opciones:
- Cambio tipo iluminación Incandescente CFL.

- Cambio tipo iluminación CFL - LED.

- Cambio tipo Tv CRT - LCD.

\subsubsection{Cambio de Habito de Consumo.}

Los habitos son patrones o costumbres en horarios $\mathrm{y}$ cantidad de tiempo donde se usan los diferentes electrodomesticos en el hogar, por tanto estan directamente relacionados con la rutina diaria de los usuarios en el hogar.

Dentro de la estrategia de cambio de hábito se adoptaron las siguientes opciones:

- Cambio temperatura nominal termostato nevera $2-4^{\circ} \mathrm{C}$.

- Eliminación consumo Stand-by de televisor y codificador durante el tiempo de no empleo.

\subsubsection{Modelado de cargas.}

Las técnicas utilizadas en la simulación de las cargas corresponden a:

- Estrategias estocásticas: Cadenas de Markov de dos estados para iluminación (OnOff) y variables aleatorias de Poisson y Exponencial para el televisor (Uso y Duración). Se escogieron de esa manera para facilitar y mejorar la ejecución del programa de cada modelo.

- Estrategias determinísticas en refrigeración.

\subsection{Composicion individual de la carga}

En este primer paso se eligieron tres tipos de carga y un restante que componen el perfil de demanda general para cada usuario modelado.

\subsubsection{Nevera.}

A la hora de simular el comportamiento de la nevera, se tuvo en cuenta el tiempo de los ciclos y frecuencia de encendido del compresor, se han tomado con base en los estudios de O. Laguerre [17][19].

La Fig. 2 entrega las mediciones de temperatura realizadas sobre la superficie de tres secciones del refrigerador durante los ciclos normales de trabajo del compresor.

La energía consumida varía según la temperatura adoptada en el termostato de la nevera, la temperatura ambiente o exterior, la capacidad en volumen utilizada por los alimentos dentro del refrigerador y el número de aperturas de la nevera durante el día. Sólo el primer y el último factor de los anteriormente mencionados se tomaron en cuenta para este modelamiento de consumo de la nevera. 


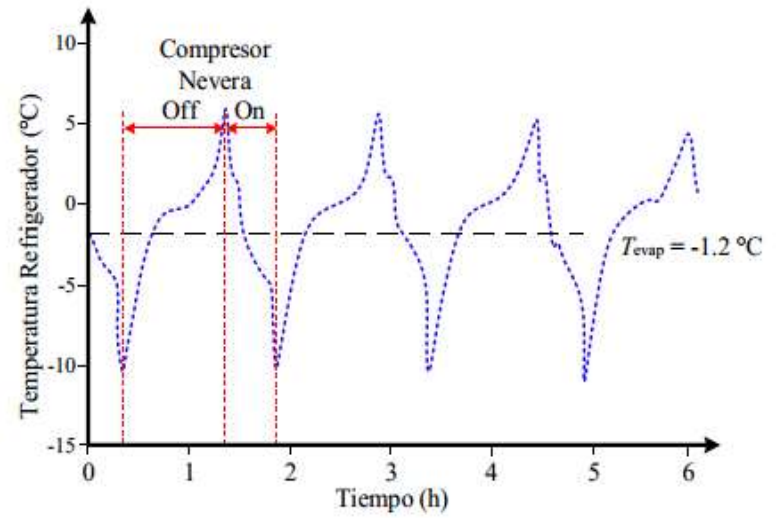

Figura 2. Ejemplo variación de la temperatura superficial del congelador de una nevera con termostato a $6^{\circ} \mathrm{C}$. Fuente: Adaptado O. Laguerre, [17].

\subsubsection{Iluminacion.}

El modelo de las fuentes luminosas se construyo bajo los siguientes supuestos:

- Siempre van a existir en cualquier residencia una cantidad mínima de 3 y una máxima de 12 fuente luminosas en cualquier combinación.

- Todos los elementos tienen probabilidad uniforme de ser utilizados y con consumo energético similar.

- Su uso no depende de la cantidad de personas en la residencia.

Se clasifican en halógenas o incandescente (60 W), CFL (Compact Fluorescent Lamps), y de tecnología LED. En este modelo aún no se tiene en cuenta la calidad de iluminación.

En la Fig. 3 se muestran tres ejemplos de modelamiento de consumo energético para las 3 tecnologías de iluminación.

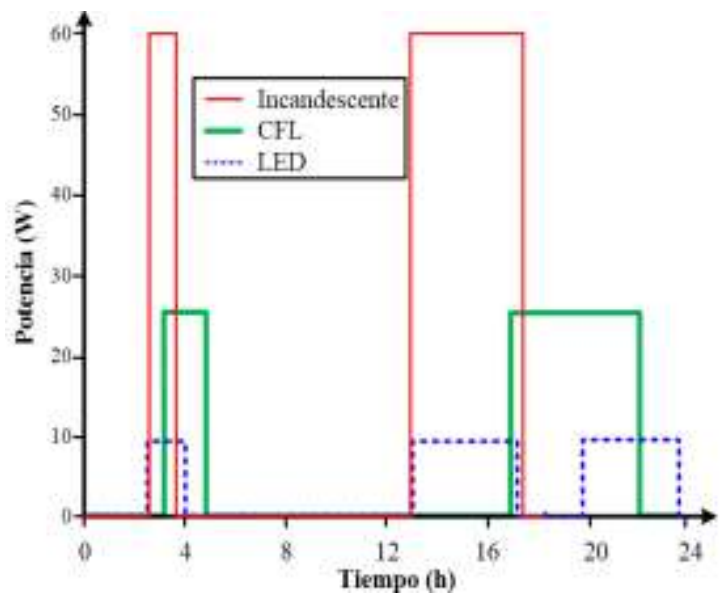

Figura 3. Ejemplos modelamiento tecnologías de iluminación. Fuente: Elaboración propia.

\subsubsection{Codificador y Televisor.}

Estos dispositivos se modelan en conjunto, debido a que son similares en horarios de uso [16].

- También son similares en características de uso como es la presencia de una energía remanente en el modo Stand-by (Disponibilidad de uso inmediato).

- El perfil de consumo se programó a través de cadenas de Markov de 3 estados, On, Off y Stand-by.

- Se ha tenido en cuenta la presencia de dos tipos de televisores, el antiguo de tubos de rayos catódicos o CRT y el nuevo de pantalla LCD.

La Fig. 4 representa ejemplos de modelamiento para un televisor nuevo y otro antiguo durante un día.

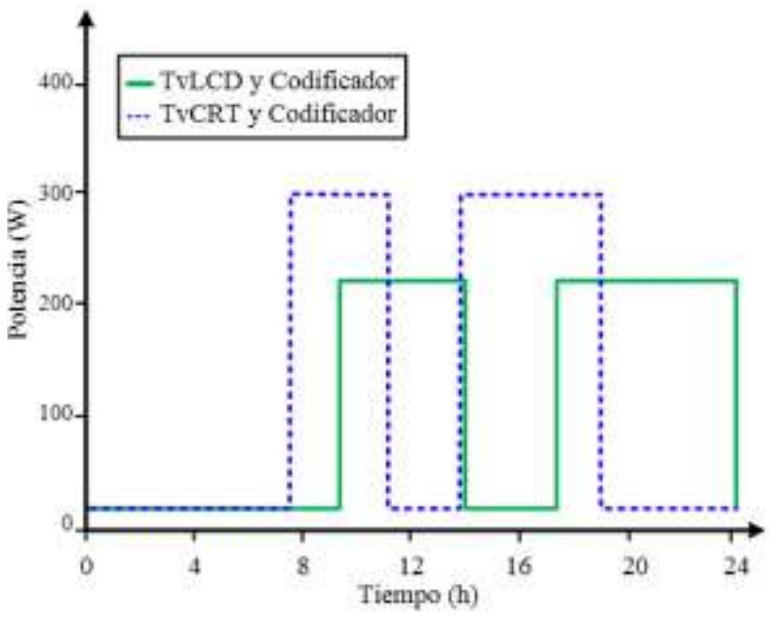

Figura 4. Ejemplos modelamiento tecnologías de televisión y codificador. Fuente: Elaboración propia.

\subsection{Combinaciones de cargas}

Debido a que es posible que se presenten diferentes combinaciones entre cantidades de dispositivos, tipos de tecnologías y hábitos de consumo, se creó una matriz base de 3 dimensiones (iluminación, refrigeración y entretenimiento) con los perfiles de consumo resultantes de todas las posibles combinaciones. Este modelo se planteó como un problema del tipo "knapsack" [20], en el cual no sólo se busca una solución óptima, sino el conjunto de soluciones posibles que cumple a cabalidad con la conformación de carga de la Tabla 2. (Ecuación (2)) y los datos de la Fig.1.

\subsubsection{Carga Base.}

Cada decil posee un conjunto de combinaciones que incluyen los diferentes usos energéticos finales como son 
iluminación, refrigeración, entretenimiento y un restante. Cada combinación encaja adecuadamente dentro de cada promedio de consumo mensual de cada decil. (Fig. 5)

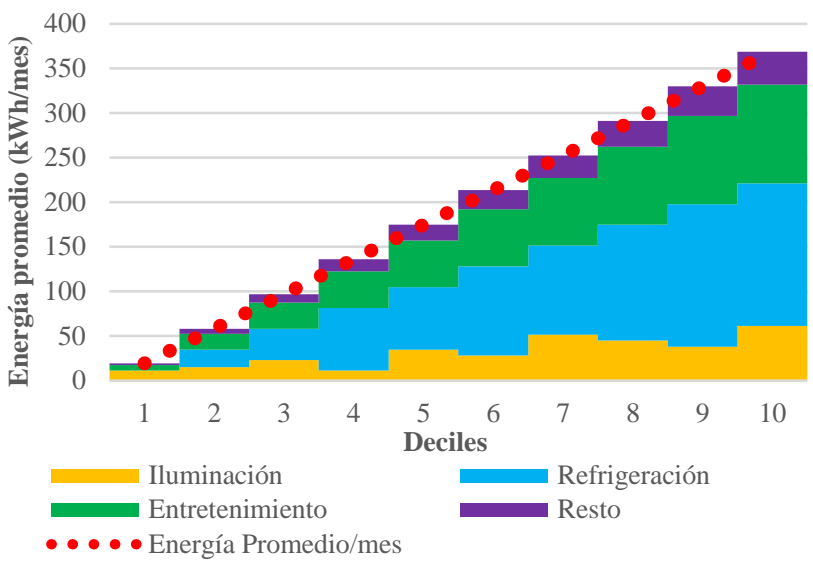

Figura 5. Ejemplo combinaciones carga base. Fuente: Elaboración propia.

2.4.2. Problema de la maleta (Knapsack Problem). Las combinaciones se realizan entre los elementos de Iluminacion, refrigeracion y entretenimiento; cada elemento anterior (energía consumida) debe ser compuesto de los datos siguientes [20], [21].

- Valor: (P) Se refiere a la Potencia nominal del elemento (Tablas 2 y 3 ).

- Peso:(W) Se refiere al tiempo de uso mensual en minutos del elemento (dependiendo de hábitos de consumo).

- Cantidad: (X) Se refiere al número de elementos para cada aplicación (Tabla 1).

- Capacidad de la mochila: (C) La energía máxima permitida para cada decil (1).

$$
E_{\text {rest }} \leq 0.1 * C_{i, j}
$$

Donde:

$E_{\text {rest }}{ }_{i, j}=$ Energía restante corresponde a otros dispositivos.

$C_{i, j}=$ Promedio Consumo Energía al mes para cada usuario $j$ que pertenece al decil $i$.

Los consumos de energía para cada uso se construyen a partir de (2).

$$
E_{i l u_{i, j}}=>E_{n e v_{i, j}}=>E_{t v c_{i, j}}=>P_{i, j} * W_{i, j} * X_{i, j}
$$

Donde:

$i=1, \ldots, 10$ decil

$j=1, \ldots, n$ Usuarios

$P_{i, j}=$ Potencia nominal del dispositivo del usuario $j$ que pertenece al decil $i$.
$W_{i, j}=$ Tiempo de uso del dispositivo del usuario $j$ que pertenece al decil $i$.

$X_{i, j}=$ Cantidad de dispositivos del mismo uso final del usuario $j$ que pertenece al decil $i$.

\subsection{Criterios de combinaciones}

Se construyen matrices de posibles combinaciones para cada grupo de usuarios según el decil al que pertenecen y sujeto a (3)[22].

$$
E_{i, j}=E_{i l u_{i, j}}+E_{n e v_{i, j}}+E_{t v c_{i, j}}+E_{r e s t_{i, j}}
$$

Donde:

$i=1, \ldots, 10$ Deciles función distribución población Bogotá

$j=1, \ldots, n$ Usuarios pertenecientes al decil $i$

$E_{i, j}=$ Energía Promedio usuario $j$ para el decil $i$.

$E_{i l u_{i, j}}=$ Energía correspondiente a iluminación

$E_{n e v_{i, j}}=$ Energía correspondiente a nevera

$E_{t v c_{i, j}}=$ Energía correspondiente a tv y codificador

$E_{\text {rest }}{ }_{i, j}=$ Energía restante respecto al promedio

Otros criterios asumidos para disminuir tiempos de procesamiento se dan en (4).

$$
E_{i l u_{i, j}} \leq 0.6 * E_{i, j} \quad E_{t v c_{i, j}} \leq 0.4 * E_{i, j}
$$

\section{RESULTADOS}

El método empleado arroja una matriz con todas las combinaciones posibles a la cual se aplican las restricciones dadas en las ecuaciones (1),(3) y (4), dando como resultado matrices de combinaciones exitosas para cada decil de la distribución de población de estrato IV en la ciudad de Bogotá.. Por ahora las combinaciones exitosas se consideran uniformemente distribuidas y por tanto con igual probabilidad de ocurrencia en la poblacion.

Cada una de esas matrices exitosas se ordena de acuerdo a la cantidad de energía consumida $\left(P_{i, j} * W_{i, j}\right)$ y cantidad de elementos $\left(X_{i, j}\right)$, con el fin de encontrar los casos (base) en que las estrategias de gestión de demanda no son aplicables, como lo son consumo minimo posible, o presencia de elementos altamente eficientes.

\subsection{Aplicación de las estrategias de gestión}

A partir del ordenamiento anterior se registran las posibilidades de gestion por cantidad de elementos (sustitución tecnológica) y por rango de consumo energetico (hábitos de consumo energetico). Los 
resultados se analizaron segun la estrategia adoptada a continuación:

Sustitución: En iluminación y entretenimiento. Los resultados obtenidos desde la aplicación de las herramientas de gestión en sustitución tecnológica, Fig. 6 , muestra una tendencia positiva en cuanto al ahorro posible en iluminación, sin embargo en entretenimiento se mantiene casi constante.

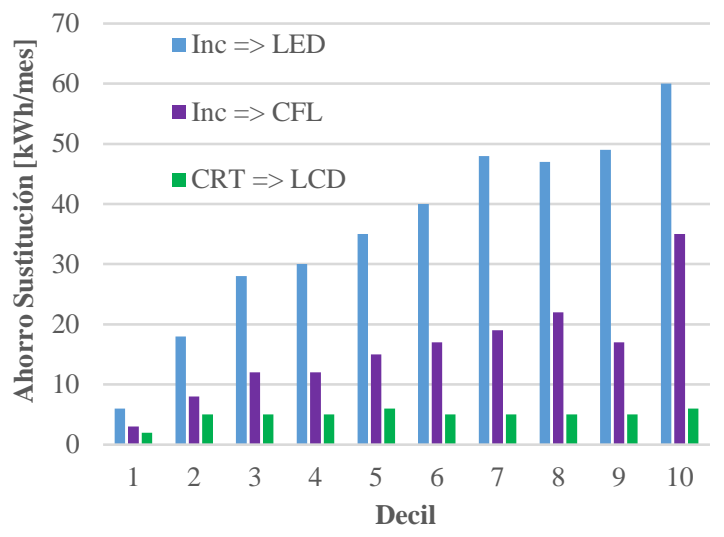

Figura 6. Sustitucion tecnológica en iluminación, de Incandescence a CFL e Incandescente a LED.

Cambio de Hábito de Consumo: en Refrigeración y Entretenimiento. En la Fig. 7 representa los ahorros posibles para los clientes que posean elementos de refrigeración e iluminación. Se denota que el decil 1 no posee ahorros en el cambio de hábito en refrigeración debido a que el consumo promedio es tan insuficiente que se deduce que no usen esta tecnología.

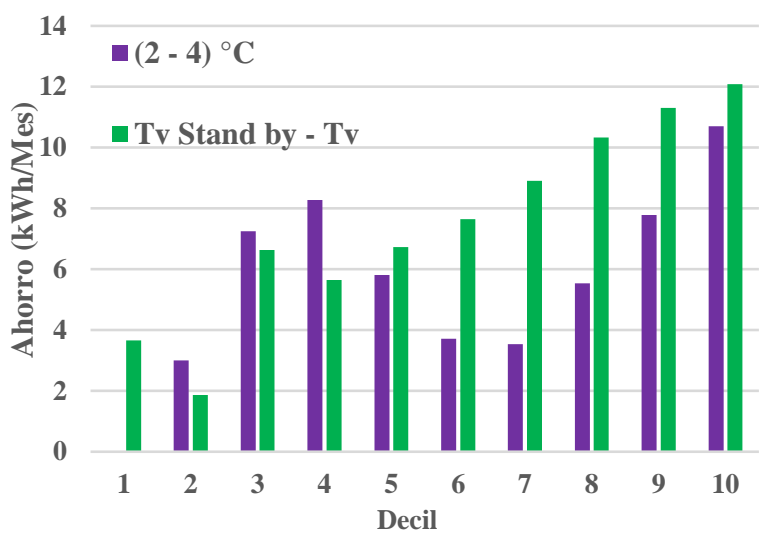

Figura 7. Cambio en la temperatura de en el reostato de la nevera y cambio de habito en televisión.

Las tendencias observadas en las Fig. 6 y 7 (pendiente positiva) reflejan una oportunidad de gestión con alto potencial de ahorro en los deciles de mayor consumo energético. En otras palabras, es más probable lograr mayores ahorros de energía por cliente en los usuarios que consumen grandes cantidades de energía.

La Fig. 8 representa el ahorro combinado usando las estrategias de sustitución en iluminación (CFL a LED, debido a que aún no es posible determinar la actual penetración de la descontinuada iluminación incandescente), entretenimiento (Tv CRT a TV LCD), y cambio de hábito en refrigeración (Cambio $\mathrm{T}^{\circ} 2$ a $4^{\circ} \mathrm{C}$ ), en entretenimiento (Stand-by a 0 Stand-by). Este ahorro combinado se expresa para cada decil en:

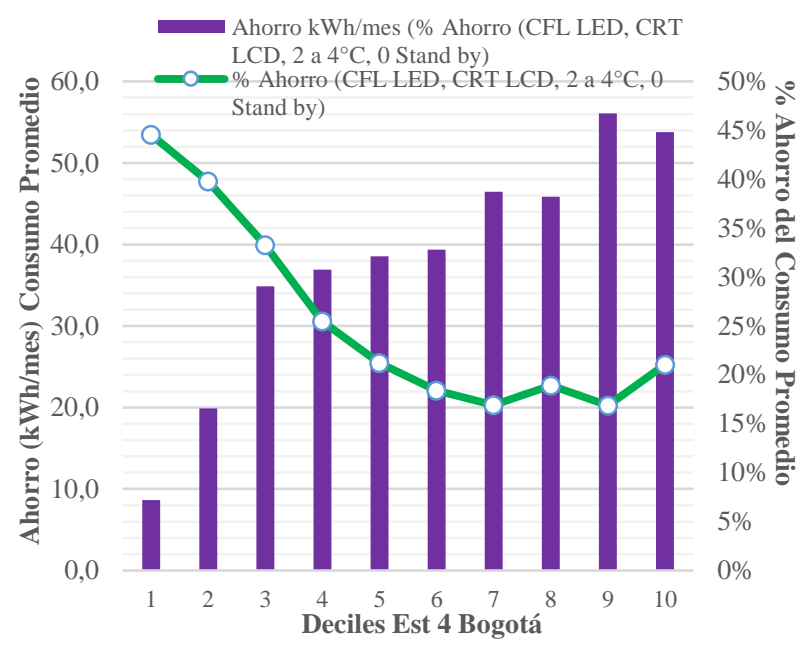

Figura 8. Ahorros combinados Sustitución y Cambio de Habito para los deciles Estrato IV de Bogotá.

-Energía factible de ahorrar cada decil (kWh/mes) eje vertical primario (izquierdo).

- Porcentaje de la energía factible de ahorrar respecto del promedio de consumo mensual para cada decil, eje vertical secundario (derecha).

Aunque la cantidad de energía que puede ahorrarse en los primeros deciles es baja, esta representa respecto a su promedio de consumo mensual un porcentaje más alto comparado con el resto de los deciles de mayor consumo.

La Tabla 4. presenta los resultados generales de la investigación discriminados para cada decil de la población modelada. Las cantidades más altas de energía para considerar oportunidades de ahorro se encuentran en sustitución. Sin embargo, el cambio de hábitos de consumo por parte de los usuarios residenciales de estrato IV en la ciudad de Bogotá, abre la oportunidad de realizar más de la mitad del ahorro en energía comparado con sustitución, con la ventaja de requerir un coste económico mucho menor. 
Tabla 4. Ahorros globales: Sustitución y cambio de hábitos.

\begin{tabular}{|c|c|c|c|c|c|c|}
\hline \multirow{2}{*}{ Decil } & \multicolumn{2}{|c|}{ Ahorro Sustitución } & \multicolumn{2}{c|}{ Ahorro Cambio Habito } & \multicolumn{2}{c|}{ Ahorro Total } \\
\cline { 2 - 7 } & {$[\mathbf{k W h} / \mathbf{m e s}]$} & $\mathbf{( \% )}$ & {$[\mathbf{k W h} / \mathbf{m e s}]$} & $\mathbf{( \% )}$ & {$[\mathbf{k W h} / \mathbf{m e s}]$} & $\mathbf{( \% )}$ \\
\hline 1 & 5 & $26 \%$ & 3,7 & $19 \%$ & 8,7 & $45 \%$ \\
\hline 2 & 13 & $26 \%$ & 10,2 & $17 \%$ & 19,9 & $40 \%$ \\
\hline 3 & 17 & $22 \%$ & 15,3 & $16 \%$ & 34,9 & $33 \%$ \\
\hline 4 & 17 & $17 \%$ & 17,7 & $13 \%$ & 52,7 & $39 \%$ \\
\hline 5 & 21 & $15 \%$ & 16,1 & $9 \%$ & 36,9 & $25 \%$ \\
\hline 6 & 22 & $13 \%$ & 17,3 & $8 \%$ & 38,5, & $21 \%$ \\
\hline 7 & 24 & $13 \%$ & 18,7 & $7 \%$ & 39,4 & $18 \%$ \\
\hline 8 & 27 & $10 \%$ & 28,1 & $10 \%$ & 46,4 & $17 \%$ \\
\hline 9 & 22 & $11 \%$ & 33,7 & $10 \%$ & 45,9 & $19 \%$ \\
\hline 10 & 41 & $8 \%$ & 36,6 & $10 \%$ & 53,8 & $21 \%$ \\
\hline
\end{tabular}

El decil 4 entrega una oportunidad de ahorro cercano a los $1.2 \mathrm{GWh} / \mathrm{mes}$ usando las estrategias aprovechadas en el estudio; le siguen los deciles $3,5,6$ y 7 con cifras que superan $500 \mathrm{MWh} / \mathrm{mes}$.

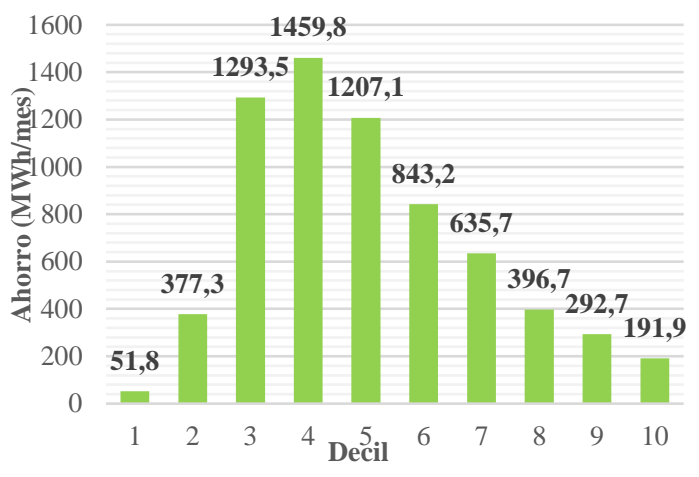

Figura 9. Ahorros totales combinando estrategias de Sustitución y Cambio de hábito junto con el número de clientes por decil para el Estrato IV en Bogotá.

\section{CONCLUSIONES}

La identificación de acciones de gestión de demanda requiere conocer el consumo de energía de un cliente y su perfil de demanda. En el presente artículo se estimó la composición de carga con base en criterios de optimización y en las alternativas sugeridas en la literatura. La cantidad de esquemas de composición de carga es enorme, aún con las aproximaciones propuestas.

Por lo anterior, se encontró que los métodos empleados para escoger la composición de carga de cada cliente representan un reto importante en la identificación de las estrategias de gestión más adecuadas. Se encontró que la cantidad de combinaciones posibles para los grupos de tecnologías propuestas es del orden de $10^{12}$ y aumenta exponencialmente si se incluyeran mas grupos. La exploración mediante métodos de Monte Carlo ofrece una alternativa computacionalmente eficiente, para trabajos futuros.

Las estrategias indicadas pueden proporcionar hasta un $19 \%$ de ahorro en energía eléctrica, sobre el consumo base. Lo anterior depende de la cantidad de estrategias adoptadas por los usuarios, y de los electrodomésticos presentes en el hogar sobre los cuales se aplicarían dichas estrategias.

A pesar de que los mayores ahorros de energía por cliente pueden lograrse en los clientes con mayores consumos mensuales, el mayor ahorro de energía agregado para todos los clientes considerados se logra entre los deciles $3,4,5$ y 6 , es decir, en los clientes con consumos entre 100 y $250 \mathrm{kWh} / \mathrm{mes}$. Lo anterior permite prever que puede lograrse un impacto mayor si se implementan estrategias de gestión en los clientes con los consumos mensuales identificados, y no en los clientes con consumos más altos y más bajos.

\section{REFERENCIAS}

[1] R. Poudineh and T. Jamasb, "Distributed generation, storage, demand response and energy efficiency as alternatives to grid capacity enhancement," Energy Policy, vol. 67, pp. 222-231, 2014.

[2] N. O'Connell, “Approaches for Accommodating Demand Response in Operational Problems and Assessing its Value," Technical University of Denmark (DTU), Kgs. Lyngby, 2016.

[3] M. H. Albadi and E. F. El-Saadany, "Demand response in electricity markets: An overview," in 2007 IEEE Power Engineering Society General Meeting, PES, 2007. 
[4] J. Torriti, M. G. Hassan, and M. Leach, "Demand response experience in Europe: Policies, programmes and implementation," Energy, vol. 35, no. 4, pp. 1575$1583,2010$.

[5] M. H. Albadi and E. F. El-Saadany, "A summary of demand response in electricity markets," Electric Power Systems Research, vol. 78, no. 11. pp. 1989-1996, 2008.

[6] O. Corradi, H. Ochsenfeld, H. Madsen, and P. Pinson, "Controlling electricity consumption by forecasting its response to varying prices," Power Syst. IEEE Trans., vol. 28, no. 1, pp. 421-429, 2013.

[7] P. Thorsnes, J. Williams, and R. Lawson, "Consumer responses to time varying prices for electricity," Energy Policy, vol. 49, pp. 552-561, 2012.

[8] H. Allcott, "Rethinking real-time electricity pricing," Resour. Energy Econ., vol. 33, no. 4, pp. 820-842, 2011.

[9] O. A. Gonzalez and A. Pavas, "Evaluación del Potencial de Respuesta de la Demanda a partir de información de Perfiles de Consumo," in V CIUREE, Congreso Internacional de Uso Racional de la Energía, Cartagena, Colombia, Feb, 2016.

[10] M. Doostizadeh and H. Ghasemi, "A day-ahead electricity pricing model based on smart metering and demand-side management," Energy, vol. 46, no. 1, pp. 221-230, 2012.

[11] S. Yousefi, M. P. Moghaddam, and V. J. Majd, "Optimal real time pricing in an agent-based retail market using a comprehensive demand response model," Energy, vol. 36, no. 9, pp. 5716-5727, 2011.

[12] N. Arghira, L. Hawarah, S. Ploix, and M. Jacomino, "Prediction of appliances energy use in smart homes," Energy, vol. 48, no. 1, pp. 128-134, 2012.

[13] J. H. Kim and A. Shcherbakova, "Common failures of demand response," Energy, vol. 36, no. 2, pp. 873$880,2011$.

[14] N. National Institute Standards and Technology, "NIST: Smartgrid." [Online]. Available: http://www.nist.gov/smartgrid/. [Accessed: 23-Aug2016].

[15] ENERGY.GOV, "Estimating Appliance and Home Electronic Energy Use | Department of Energy." [Online].Available:http://energy.gov/energysaver/estima ting-appliance-and-home-electronic-energy-use.

[Accessed: 23-Aug-2016].
[16] L. L. Gracia and L. C. Torrecillas, "Guía sobre Consumo Energético de Aparatos Domésticos," Zaragoza, 2009.

[17] O. Laguerre, "Heat transfer and air flow in a domestic refrigerator," Math. Model. Food Process. Mohammed M. Farid, vol. 474, pp. 445-474, 2010.

[18] Instituto colombiano de normas técnicas y certificación, Eficiencia energética en artefactos refrigeradores, refrigeradores-congeladores y congeladores para uso doméstico NTC 5020. Bogotá DC. Colombia, 2014, p. 56.

[19] H. Park, M. Ruellan, N. Martaj, R. Bennacer, and E. Monmasson, "Generic thermal model of electrical appliances in thermal building: Application to the case of a refrigerator," Energy Build., vol. 62, pp. 335-342, 2013.

[20] S. Martello and P. Toth, Knapsack problems: algorithms and computer implementations. John Wiley \& Sons, Inc., 1990.

[21] H. Kellerer, U. Pferschy, and D. Pisinger, Knapsack Problems, 1st ed. Berlin, Heidelberg: Springer-Verlag Berlin Heidelberg, 2004.

[22] F. Sandoya, "El Problema de la Mochila, Complejidad, Cotas y Métodos de Búsqueda Eficientes," vol. 12, no. 2, pp. 43-51, 2014. 